\title{
VASCULITIS DAMAGE INDEX AND C-REACTIVE PROTEIN AS MAIN PREDICTORS OF INFECTION IN ADMITTED SYSTEMIC VASCULITIS PATIENTS
}

Luma Mendes Brito ${ }^{1, *}$, Felipe Mendonça de Santana ${ }^{1}$, Rodrigo da Silveira Vasconcelos ${ }^{1}$, Carlos Emilio Insfran Echauri ${ }^{1}$, Lorenza Rosa Silvério Scomparin ${ }^{1}$, Rafael Pontes Andreussi ${ }^{1}$, Lissiane Karine NoronhaGuedes ${ }^{1}$, Rosa Maria Rodrigues Pereira ${ }^{1}$, Maira Luciana Marconcini de Lacerda ${ }^{1}$

1. Universidade de São Paulo, São Paulo (SP), Brasil.

*Corresponding author: lumamendesbrito@gmail.com

\section{BACKGROUND}

Systemic vasculitis encompasses a heterogeneous group of diseases distinguished by the presence of an inflammatory response within the vessels wall. Its treatment often requires the use of immunosuppressive. Hence, infections are fairly common in vasculitis patients. However, discerning acute infection from disease relapse is not straightforward. We searched for clinical differences between vasculitis patients admitted due to severe infections and those admitted due to other conditions.

\section{METHODS}

A cross-sectional study was performed using standard electronic medical records of a rheumatology tertiary center. Data from consecutive inpatients admitted on rheumatology clinics from July 2014 to July 2019 were revised. Those with confirmed primary systemic vasculitis were included. Forty patients (most of them with granulomatosis with polyangiitis) were considered eligible for analysis, comprising 55 admissions. The patients were classified regarding the presence or absence of infection at entry. Data collected during hospitalization was used to ascertain whether the baseline clinical condition was indeed confirmed as infection, including cultures and radiologic findings. Baseline variables were compared between patients admitted due to infection (Infection group) and those admitted for other conditions (noninfection group). The infection and the noninfection groups consisted of 17 and 38 admissions, respectively. Respiratory infections were most common (8 out of 17). The noninfection group comprised mainly of patients admitted due to vasculitis activity. Chi-square test and Fisher's exact test were used for categorical variables and Welch t-test, Student's t-test and Mann-Whitney test for quantitative variables, where appropriate. The alpha level was set at 0.05 . Multiple logistic regression was performed using a backward stepwise selection using Wald test to find which variables best predicted the presence of infection. All tests met their respective required assumptions.

\section{RESULTS}

Patients admitted due to infection had significantly higher levels of creatinine (mg/dL) [1.23 (IQ 1.00-3.70) vs. 0.80 (IQ 0.68 1.17), $\mathrm{p}=0.01$ ), of $\mathrm{C}$-reactive protein ( $\mathrm{mg} / \mathrm{L}$ ) [49 (IQ 37-73) vs. 14 (IQ 7-32), $\mathrm{p}=0.02]$, a higher baseline vasculitis damage index (VDI) [5 (IQ 5-7) vs. 4 (IQ 3-5) p=0.02) and were more frequently ANCA-positive [11/17 (64.7\%) vs. 12/38 (31.6\%), $p=0.04]$ and on prophylactic oral trimethoprim/sulfamethoxazole (TMP/SMX) [7/17 (41.2\%) vs. 3/38 (7.9\%), $p<0.01]$. At multivariate analysis, the independent predictors of infection were VDI $[\mathrm{OR}=1.83(95 \% \mathrm{Cl}=1.24-2.72), \mathrm{p}=0.002]$ and values of $\mathrm{CRP}$ in the higher tercile $[\mathrm{OR}=51.30$ ( $95 \% \mathrm{Cl}=2.93-897.00), \mathrm{p}=0.006]$. No significant differences regarding age, sex, cumulative dose of glucocorticoid in previous year, lifetime cumulative dose of cyclophosphamide, immunobiological agent use, days of admission, BVAS, leucocytes and serum gamma globulins were identified. Death was observed in two patients with infections.

\section{CONCLUSIONS}

Infection was closely related with CRP levels and previous damage of disease but not with activity scores and previous treatments at admission. Although an increase in CRP may be seen in disease activity, infection does it more consistently. These predictors may aid the treating physician in differentiating disease activity from intercurrent infection. Further studies are necessary to confirm the role of these findings. 